\title{
BSDF Reconstruction of Inks and Paints for Light Guiding Plates
}

\author{
Vadim Sokolov ${ }^{1,2}$, Igor Potemin ${ }^{1}$, Dmitry Zhdanov $^{1}$ and Boris Barladian ${ }^{2}$ \\ ${ }^{1}$ ITMO University, 49 Kronverksky Pr., St. Petersburg, 197101, Russia \\ ${ }^{2}$ Keldysh Institute of Applied Mathematics, Russian Academy of Sciences, Miusskaya sq. 4, Moscow, 125047, \\ Russia
}

\begin{abstract}
The work is devoted to the investigation of the best and reasonable methods to reconstruct the scattering properties of inks and paints widely used in modern electronic devices containing various light guiding plates and fibers. Typically scattering properties for inks have a volume scattering nature however it can be complicated with surface scattering because of the microroughness of ink layers. Both the volume and surface scattering reconstruction are considered in the given paper. Typically scattering properties of inks are described with bidirectional scattering functions (BSDF) and the BSDF is required from the internal side of the material of light-guiding elements. It cannot be measured directly because neither detector of the measuring device nor the light source cannot be placed inside the material. The only way to solve the problem of BSDF extraction for such cases is to build computer models of volume and surface scattering for inks and then with optimization procedures reconstruct parameters for these models which can provide surface scattering close enough to scattering of real samples of inks and paints. Light propagation in ink media is very complex requiring a long calculation time. As a result, the selection of an effective optimization procedure is the key point of the task. Several more typical real samples with printed inks are selected for investigation. The achieved results of BSDF extraction show very good agreement with data obtaining by real measurement. The comparison is based on numerical comparison of light scattering of ink samples as well as its visual appearance.
\end{abstract}

\section{Keywords}

Ink, Bi-Directional Scattering Function, BSDF, Volume Scattering, Surface Scattering, Light Guiding Systems, Rendering, Visualization

\section{Introduction}

The light-guiding plates with complex scattering properties are widely used in modern optical systems. Examples of such devices can be light guiding plates for LCD, luminaires light-guiding elements in cars dashboards, different types of measuring devices. The ink and paint layers are used in such devices to provide desired complex angular and spatial light distributions. The accurate simulation of such layers is complicated because of the difficulty to describe light transformation on them. A more direct way is to describe the optical scattering properties of ink or paint with bi-directional scattering functions (BSDF). The function can be obtained with measurements however most cases it is a complex, expensive, and sometimes impossible task. Figure 1 shows several standard schemes of ink usage which eliminates evident problems related to measurements of ink scattering properties. There are three options for printing ink on a transparent dielectric plate. In the first case, ink is printed to the top plate face, in the second case - to the bottom face, and in the last case, ink is printed for both plate sides. It is evident in all cases to provide correct light propagation inside of light guiding plate it is necessary to define light scattering function inside of material as for reflectance as well for transmittance. However

GraphiCon 2021: 31st International Conference on Computer Graphics and Vision, September 27-30, 2021, Nizhny Novgorod, Russia EMAIL: sokolovv1969@gmail.com (V. Sokolov); ipotemin@yandex.ru (I. Potemin); ddzhdanov@mail.ru (D. Zhdanov); bbarladian@gmail.com (B.Kh. Barladian)

ORCID: 0000-0002-1719-5102 (V. Sokolov); 0000-0002-5785-7465 (I. Potemin); 0000-0001-7346-8155 (D. Zhdanov); 0000-0002-23912067 (B.Kh. Barladian)

$\begin{array}{lll}\text { (c) (i) } & \text { (C) } 2021 \text { Copyright for this paper by its authors. }\end{array}$

Use permitted under Creative Commons License Attribution 4.0 International (CC BY 4.0).

CEUR Workshop Proceedings (CEUR-WS.org) 
precise measurements inside of material are not possible because internal total reflections of light which will add parasitic light to measured scattering function apart from it is not possible to place detector of measuring device inside of the material.

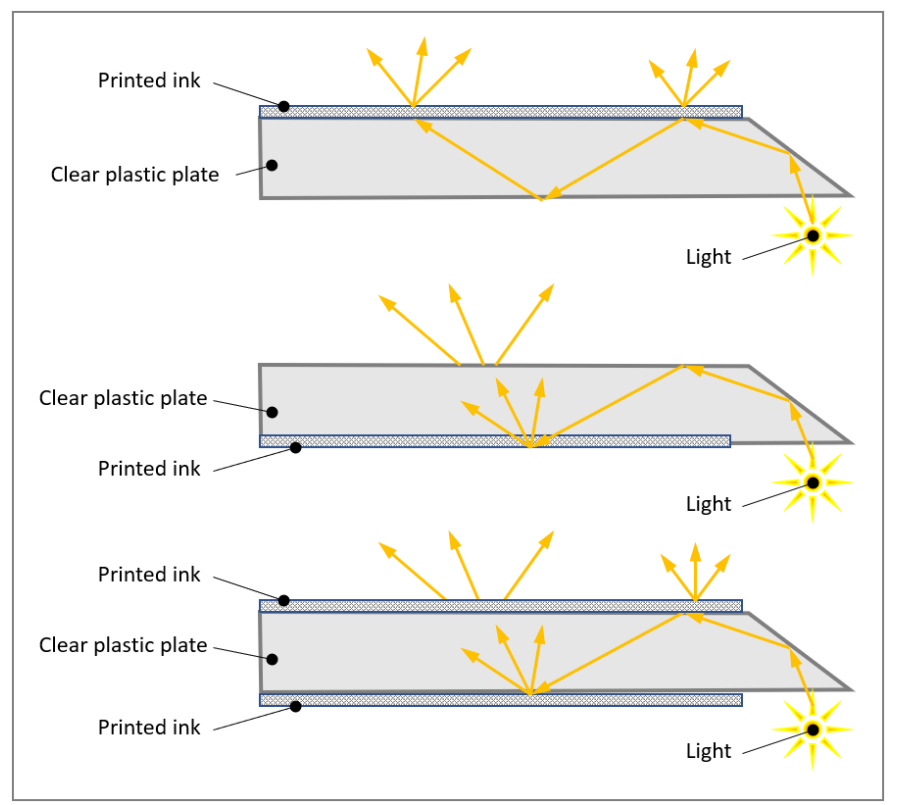

Figure 1: Examples of ink usage in different light guiding plates schemes

The problems related to measurements mentioned above practically exclude direct BSDF measurements to obtain scattering properties for ink materials. To solve the problem typically computer model of scattering is used to describe volume and surface scattering for ink and paint layers. However, building an analytical computer model of volume or/and surface scattering it is required detailed information about media and surface properties and structure which as a rule are absent. In most cases, there is a material sample, for example in a form of a plane-parallel plate, but the parameters of the individual ink components such as information about ink pigment (particle) concentration, shape, optical properties of ink binder, height distribution of ink layer surface and other elements of the ink layer are absent. These parameters are extracted with help of optimization procedures. The purpose of the optimization procedure, in this case, is to obtain the BSDF of the whole sample maximally close to the measured BSDF. Note measurements of BSDF for the whole sample without separation of ink properties from a plate to which ink is printed is a rather trivial and simple task. After the definition of ink parameters, it is possible to calculate BSDF of the ink layer separately from the plate. In computer modeling, it is not a problem to place as a source of illumination as well as light detectors inside of material required for ink BSDF extraction. This process generally is described in [1]. The investigation in the given work is deeper and based on BSDF extraction of real samples with different inks characteristics including colored samples and some conclusions are different because of more detailed and practical investigations. Apart from comparison with measurements is realized not only on a numerical level and with help of visualization. The separate part of the work is an examination of different optimization methods used for BSDF extraction and the development of recommendations to select a more effective optimization method for BSDF extraction for inks and paints.

\section{Methods to describe volume and surface scattering for inks and paints \\ 2.1. Volume Scattering model}

There are a lot of methods for the reconstruction of BSDF for Volume Scattering media [2-7]. Most of them are used in computer graphics and in lighting simulations, nevertheless, they are not applicable in tasks connected with diffuse and transparent materials in the case physical accurate modelling is required. Apart from most of them are not fully described or used only with specific software. In this 
article it is used a one of more general methods close to one described in [1]. The Figure 2 shows simplified scheme of ink layer.

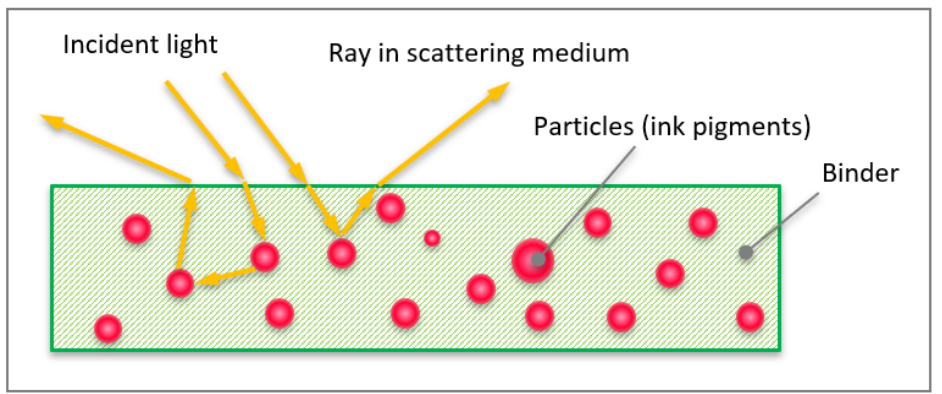

Figure 2: The simplified ink structure

The figure presents in very general terms the scattering mechanism in a heterogeneous ink medium. Light scattering in a binder medium occurs when light propagating inside collides with scattering agents (particles) having optical properties different from the binder (refractive index, dispersion, etc.). The nature of this scattering is stochastic and most often the form of angular scattering is closer to diffuse and depends on many factors, such as the size of particles, their shape and size, particle concentration, and optical properties. The size of particles (ink pigments) is usually very small and comparable with the wavelength of light. An accurate determination of light scattering on such small particles can be modeled using wave optics only. However, purely wave solutions are extremely complex (they require colossal computational resources and memory) and, as a rule, are not used in general programs intended for optical modeling. Usually, mixed, hybrid approaches using both ray and wave optics are used for the calculation, see figure 3 .

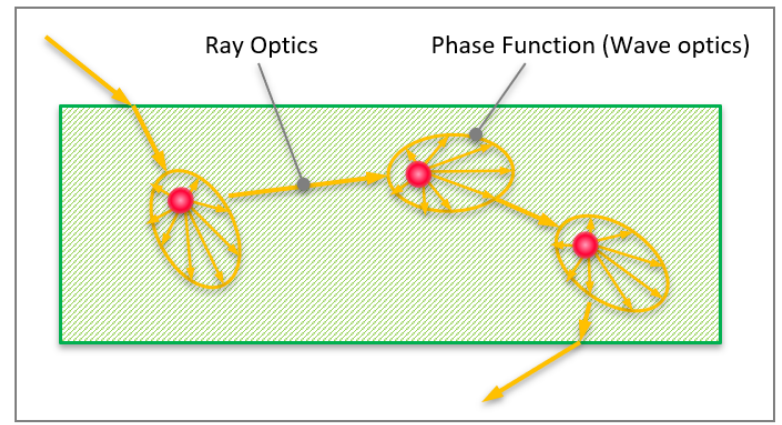

Figure 3: The usage of ray and wave optics to simulate ray propagation in ink medium

The propagation of light between the particles is determined using statistical ray tracing methods based on the average path length of the beam with the bulk medium, and the light scattering is determined using the phase scattering function (angular intensity). The form of this function is determined using the wave theory of light propagation. There are two main and well-known methods for calculating the phase scattering function. The first assumes that the shape of the scattering particles is spherical. In this case, the Mie or Rayleigh models are used to calculate the phase function, depending on the size (diameter) of the scattering particles. If the particle size is much less than the wavelength, Rayleigh scattering is used and if the particle size is commensurate with the wavelength, then the Mie theory is used. From the point of view of the problem within the framework of this work, we are primarily interested in the input parameters with which the Mie / Rayleigh model operates. These parameters are shown in the Figure 4. 


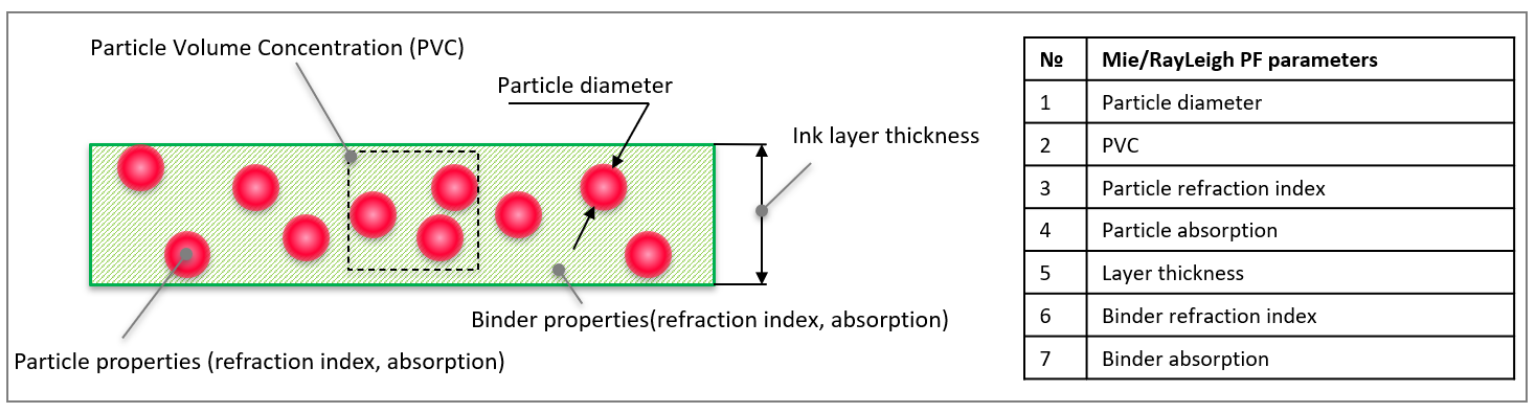

Figure 4: Volume Scattering parameters according to Mie/RayLeigh model

The second widely known method for determining the phase function is the Henie-Greenstein analytical function, which is widely used in light scattering calculations. The Henyay-Greenstein (HG) phase function is defined by the following formula:

$$
P_{H G}(\theta)=\frac{1}{4 \pi} \frac{1-g^{2}}{\left[1+g^{2}-2 g \cos (\theta)\right]^{3 / 2}}
$$

where $g$ is the anisotropy factor of the Henyay-Greenstein phase function $\theta$, - defines the angle between the incident and the scattered beam. To fully define this model, there are two more parameters describing light scattering on particles:

- Scattering - the fraction of the energy of a parallel light beam diffusely scattered over $1 \mathrm{~mm}$ path in a medium containing these particles with defined volumetric concentration.

- Absorption - the fraction of the energy of a parallel beam of light absorbed (converted into heat) per $1 \mathrm{~mm}$ path in a medium containing these particles with a certain volumetric concentration (PVC)

It should be noted that the last two parameters are defined in the format of the Lumicept software package used for research in this work [8]. The full list of parameters for Henyay-Greenstein model is shown in the Figure 5.

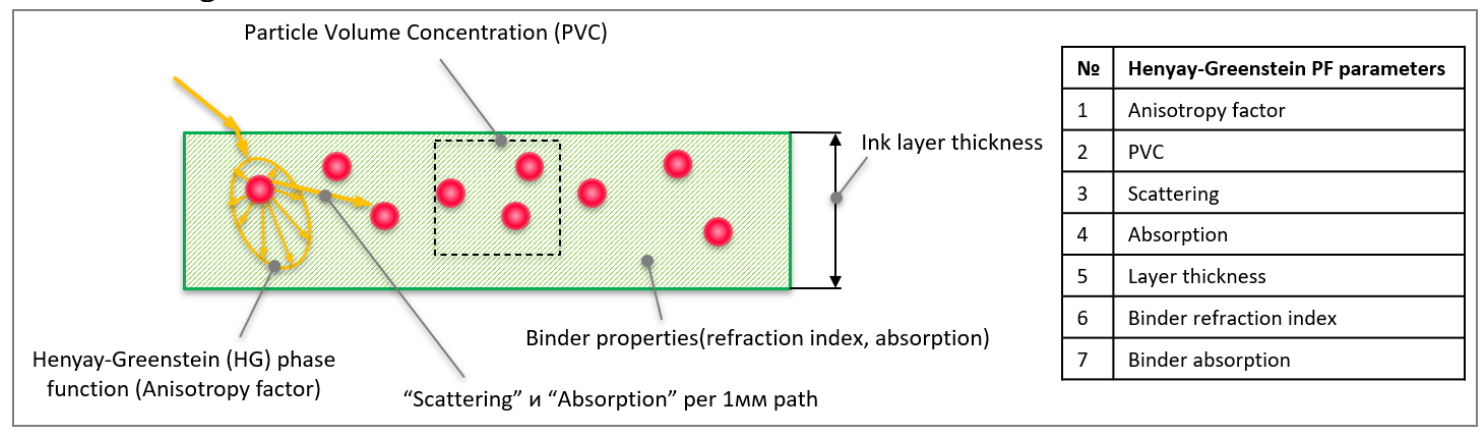

Figure 5: Volume Scattering parameters according to Henyay-Greenstein model

In the given paper both Mie/Rayleigh and Henyay-Greenstein models are used for BSDF reconstruction, the results of the investigation and conclusions about optimal model choice are presented below.

\subsection{Surface Scattering model}

For some samples ink and paint layers have rough surfaces. In the case the application of the single Volume Scattering model is not sufficient for accurate BSDF reconstruction. Thus, an addition of some surface scattering model for rough surface is required. The problem is investigated in the work. There are a lot of approaches as analytical as well numerical to reconstruct BSDF properties of rough surface scattering properties, some overview and description of the different approaches is presented in [9-11]. A numerical approach ("Normals" method) is applied. The method is described detailly in [9]. It was selected mainly because of its simplicity. The modeling of Volume Scattering itself is not trivial task and it is preferable not to complicate it with additional parameters. It is reasonable to suppose what 
main influence to scattering occurs because of heterogeneous media of ink so it is not required to simulate surface scattering with high accuracy. "sigma" parameter and "Cauchy" normal distribution has been selected for extraction BSDF for ink sample having surface with roughness.

\section{Scheme of BSDF reconstruction}

The base idea of BSDF reconstruction is not new and, for example, it can be examined in $[1,9,11]$ papers, however there are some details in simulation of Volume Scattering which it is preferable to consider with the purpose to accelerate process of BSDF extraction.

1. It should be noted that volume scattering uses wave optics (at least partially), which means that spectral calculations are required to correctly simulate the propagation of light in media with volume scattering. In addition, the propagation of light in such environments is quite complex and often with long ray paths. In other words, light calculations are rather slow, many times slower than for objects with surface scattering (for example, in comparison with rough surfaces).

2. The number of parameters describing volume scattering is much larger, at least in comparison with the parameters describing a rough surface, which in some cases have to be added to the optimization process of scattering reconstruction if the sample with volume scattering has working rough surfaces.

3. Often scattering media have color, which significantly complicates the process of BSDF reconstruction, since one has to use the spectral representation of the parameters used in the reconstruction.

4. Most of the parameters have different ranges of acceptable values, which is an unfavorable factor for use in most optimizers.

5. Often measured data (BSDF of the whole sample) which is used as objective function for optimization process contains mirror components (for example, brightness peaks when measuring reflective ink from the side of a substrate with a smooth surface) that can negatively affect the optimization process.

These specificities were considered in optimization scheme used for BSDF reconstruction. The scheme is shown in Figure 6.

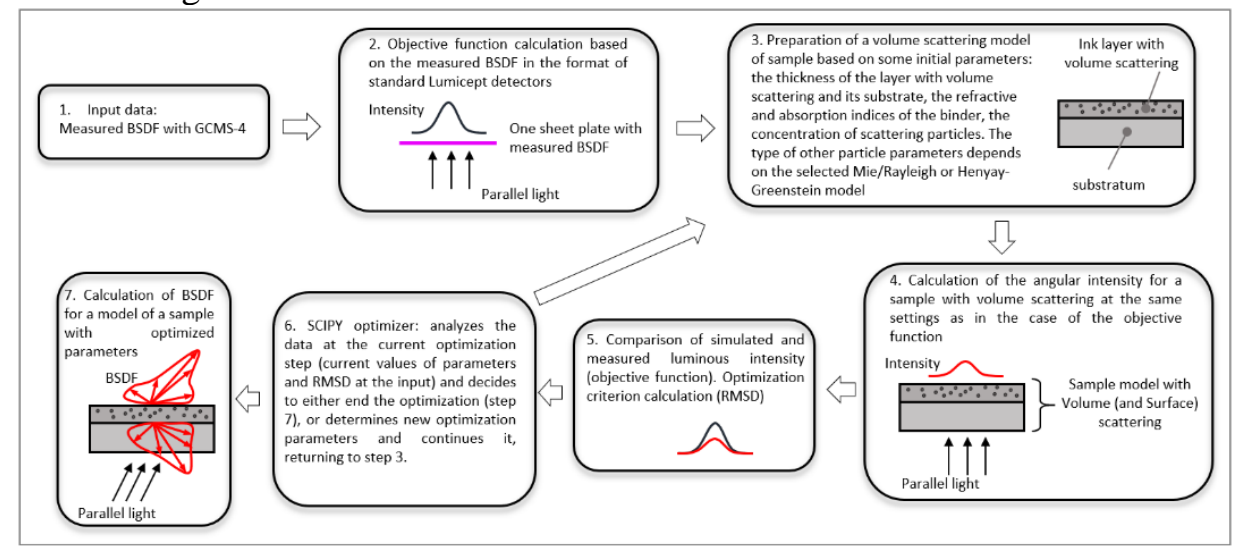

Figure 6: Optimization scheme of BSDF reconstruction for the ink

Optimization scheme presented in Figure 6 is quite typical for BSDF reconstruction:

1. The only input measured data for BSDF reconstruction process on the volume scattering layer is BSDF measured with a GCMS-4 spectroradiophotometer [12]. Ideally, for a more accurate reconstruction process, it is better to simulate a computer model of the measuring device, as it is described in [13] paper. However, calculations of volume scattering on a real rather complex model of the device take a very long calculation time. Another important point is the fact that, as a rule, media with volume scattering have more or less diffuse scattering, which makes the influence of the parameters of the measuring device (for example, angular and spatial resolution, deviation of the real gonio-diagram of the lighting system from an ideal parallel light beam, the position of the receiving system ...) not so significant. 
2. Considering factors mentioned just above, a simplified approach was used in the optimization process to calculate the objective function: The measured BSDF was assigned to an infinitely thin ("single-layer") surface. This surface was illuminated with parallel light. And then the angular light intensity was calculated in Lumicept [8] using the angular detectors standard for the program. The actually measured BSDF is simply recalculated on a more simplified scheme. Despite of the simplification, more important measuring parameters such as angular resolution were maximally matched to the measurement setup to minimize averaging errors. The recalculated BSDF (angular transmission/reflection) was used as an objective function in the reconstruction process. The calculation of BSDF for the model with volume scattering is carried out according to a similar scheme with absolutely the same parameters of illumination and light detection. Experience shows that in most cases it is sufficient to restrict one or two illumination angles close to the normal; it should also be remembered that measurements for large angles of incidence are usually not accurate, and it is better not to use them in the reconstruction process.

3. At the third step of the reconstruction process, a computer model of the sample with a scattering medium is constructed. The choice of initial parameters depends on the chosen volume scattering model (Mie/Rayleigh and Henyay-Greenstein).

4. For the constructed model of the ink sample, BSDF is calculated under the same conditions as for the measured BSDF.

5. After BSDF calculation it is compared with BSDF calculated on the base of the measured one and the root mean square deviation (RMSD) is calculated between these BSDF. Note that RMSD can be specified in both absolute and relative forms, in absolute shape:

$$
R M S D_{\text {absolute }}=\sqrt{\frac{\sum_{i}\left(I_{m i}-I_{m s}\right)^{2}}{n}}
$$

and in relative shape:

$$
R M S D_{\text {relative }}=\sqrt{\frac{\sum_{i}\left(\frac{I_{m i}-I_{s i}}{0.5\left(I_{m i}+I_{s i}\right)}\right)^{2}}{n}}
$$

Here $I_{m}$ is the measured intensity and $I_{s}$ is calculated. Index $i$ means the value of the intensity for a defined direction of observation, illumination, and wavelength in case the spectral values are used for evaluation. If the color is not important (samples are white) then the intensity is calculated in the photometric coordinate system. In the developed methodology, all directions of observation (in the range of $\pm 90^{\circ}$ ) are included in the calculation of RMSD and optionally, any direction of illumination. To speed up the optimization process, one or two directions of illumination close to the normal are usually used. In the case of diffuse scattering and in the absence of specular peaks, it is convenient to use the absolute RMSD formula; in the presence of brightness peaks, the relative RMSD is preferable. In the latter case, it is better to convert intensity values to a logarithmic scale (to reduce the parasitic influence of the brightness peaks.

6. The calculated optimization criterion (RMSD) is an input parameter for the SCIPY optimizer, which, based on this criterion, decides to either end the optimization process or continue it. In the latter case, the optimizer calculates new optimization parameters, and the process repeats from the third step. It is important to note here that the parameters of volume scattering have different dimensions - the range of variation, for example, if the refractive index changes from $1.4-2.5$, then its imaginary part, which describes the absorption, changes in the range 1e-3 - 1e-7, this is an unfavorable factor for optimizers. To eliminate this problem, you can enter weighting coefficients of the influence on each parameter, but this will lead to additional manual work, which we would like to minimize. In the implemented method, it was proposed to bring all optimization parameters to a single range from 0 to 1 . In other words, each parameter involved in the optimization process is converted to a unit interval when entered into the optimizer and then returns back to its natural value when constructing an optical model of a medium with volume scattering. As shown by numerous 
experiments, this approach gives good convergence. The question of choosing the optimal optimization method will be considered separately.

7. After completion of the optimization, up to reaching the goal or other criteria for stopping the optimization process (the maximum number of iterations, for example), a model of the sample with volume scattering is prepared and it is based on the parameters of the best optimization step and, using a special plug-in, BSDF is calculated for the layer with volume scattering, as shown in Figure 7.

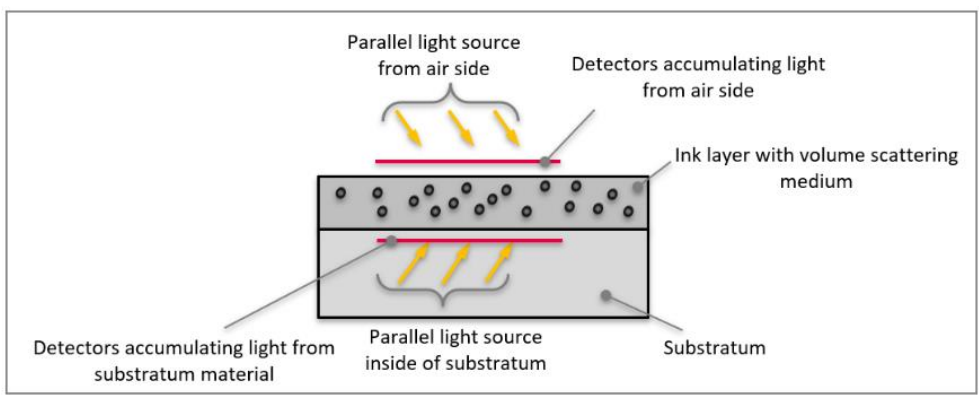

Figure 7: Scheme of ink BSDF calculation

\section{Models for verification of reconstructed BSDF for inks}

\subsection{Model for quantitative BSDF comparison with measured data}

To compare scattering light obtained with measured BSDF versus computer model of ink the following scheme is used: The volume scattering layer is alternately illuminated with parallel light at different angles of incidence from both sides of the scattering medium (from the side of the air and the filler material). For each lighting condition, the angular distribution of light is recorded with the help of special detectors (receivers) that register the angular distribution of light, which is converted into the distribution of the luminance factor and collected in BSDF - four components in the most general case describing the scattering of reflected and refracted light from both sides of the scattering layer, which can be replaced by a single-layer (infinitely thin) surface with BSDF, see Figure 8 .

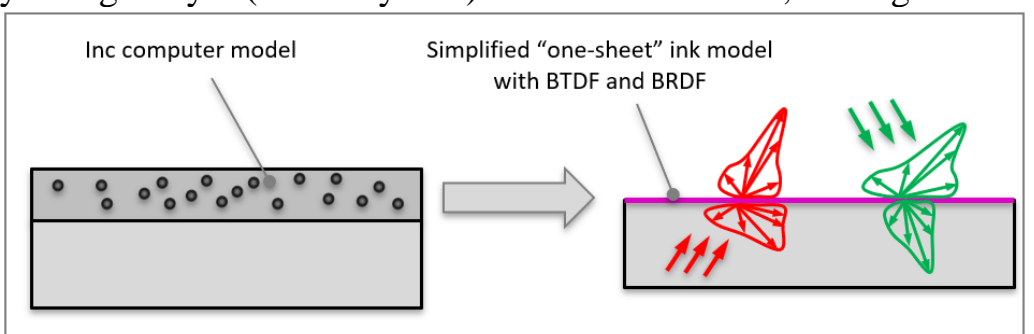

Figure 8: Simulation of ink scattering medium with BSDF on "one-sheet" model

At the final step, after ink BSDF simulation, a model of the sample with a "one-sheet" surface is built to which the reconstructed BSDF is assigned. Then the angular transmission and/or reflection of the entire plate is calculated. Then a similar calculation is started for BSDF measured with GCMS-4 spectroradiophotometer. These results are presented in the shape of a single graphs in the next chapters.

\subsection{Model for visual BSDF verification}

To visualize the reconstructed ink BSDF, a scheme like to real models aimed for color investigation is used. The model of special measuring box like JUDGE-II by X-Rite has been prepared. It has surfaces close to diffuse and several luminescent tube lamp emulating daylight. The several simplified objects (plate, sphere and torus) are placed into the measured box. On the surfaces of these test objects either the reconstructed BSDF or its analog measured with GCSM-4 is assigned. Then with the help of a special simulator ("Path Tracing" rendering available in Lumicept [8]) the images are synthesized. Note that in the case of the measured BSDF, the models of all samples are simple single-layer surfaces with the assigned measured BSDF, see Figure 9 (a), in the case of the reconstructed BSDF, solid scheme of 
test objects is considered, see Figure 9 (b), where all test objects have two surfaces. Purely "Fresnel" properties are assigned to the outer surface and reconstructed BSDF is attached to the internal surfaces. Such solid model is created because it is maximally close to task aim - define ink scattering properties (BSDF) from substratum side, see Figure 1.

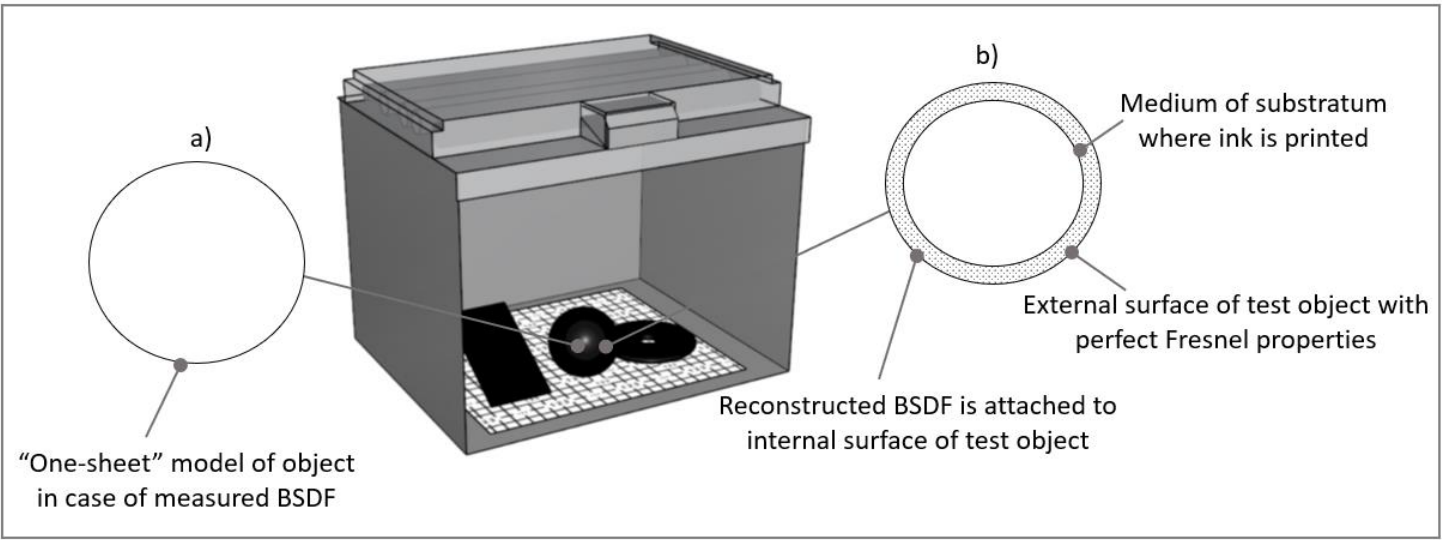

Figure 9: Models to visualize reconstructed and measured BSDF for ink samples.

\section{Description of ink samples}

Three samples have been selected for investigation. They are marked as "sample \#1", "sample \#2" and "sample \#3" Their simplified models, input data are presented in the figure 10.

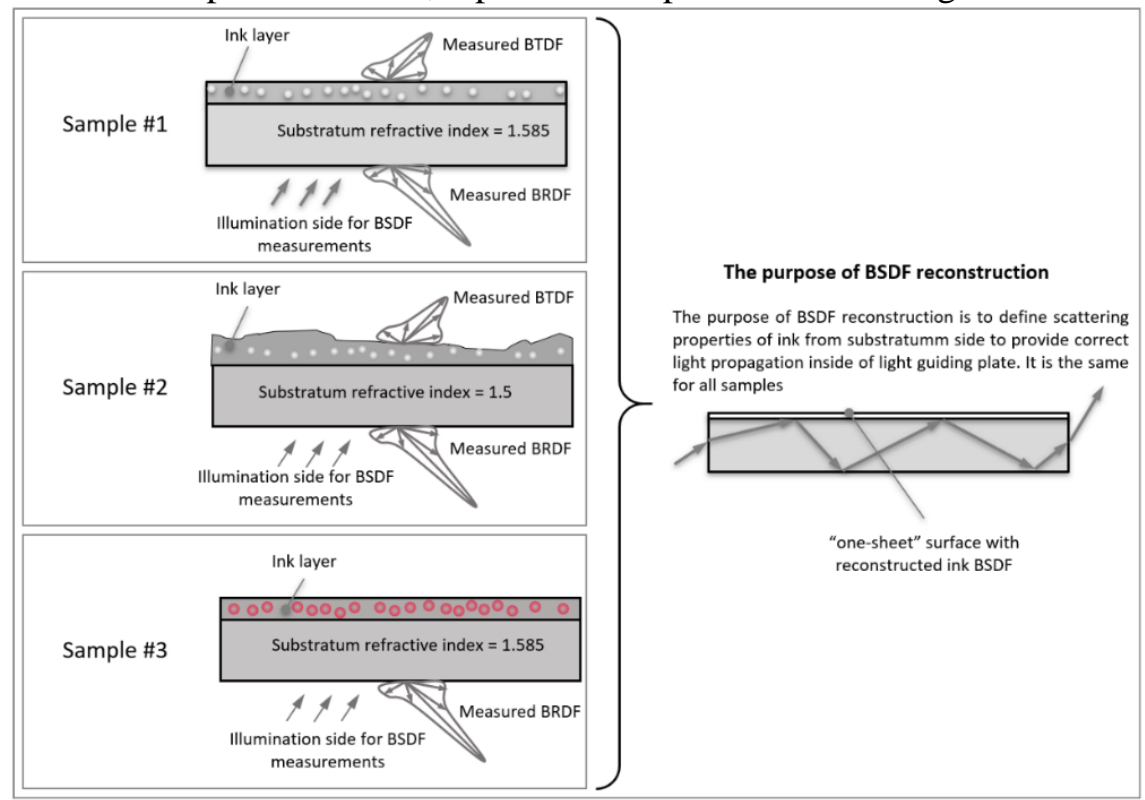

Figure 10: Description of ink samples used in the investigation

1. Description of sample \#1: It is a white ink/paint with reflective properties close to diffuse. The working surface of the ink is on the side of the substrate (inside the dielectric material). In other words, a direct measurement of such a BRDF is impossible. All surfaces of the sample (scattering layer and substrate) are smooth (polished). From the known data, there is the refractive index of the substrate and BSDF measured with GCMS-4. It is measured for both reflected (BRDF) and transmitted light (BTDF) under illumination from the side of the substrate, ink layer thickness equal $0.063 \mathrm{~mm}$. Features: The presence of specular reflection from the substrate, which is parasitic for the BSDF reconstruction.

2. Description of sample \#2: This sample is similar to the first one in terms of the type of measurements (BRDF, BTDF) under illumination from the side of the substrate. The goal is also the same to restore the ink BRDF from the side of the substrate. In addition to the measured BSDF, a 
substrate material (acryl) with a refractive index close to 1.5 is known. Features: The presence of specular reflection from the substrate, microroughness on the upper surface of the ink.

3. Description of sample \#3: This sample is the most difficult case to restore scattering properties, ink of sample is colored and it is necessary not only to reconstruct the shape of the reflected light scattering, but also the desired color. The situation is simplified by the fact that the ink in a real device is illuminated in a rather narrow spectral range from 570 to 630 nanometers, thus the process of BSDF reconstruction can be limited to a narrow spectral range in comparison with white samples. The working surface of the paint is on the side of the substrate (inside the dielectric material). All surfaces of the sample (scattering layer and substrate) are smooth (polished). From the known data, there is the refractive index of the substrate and BRDF measured with GCMS-4 spectroradiophotometer under illumination from the side of the substrate.

\section{Simulation results}

\subsection{Results of sample \#1 reconstruction}

It should be noted that this sample was the simplest of the investigated set, where the scattering properties of the ink are very close to the ideal (Lambertian) diffuser. Since the parameters of the internal structure of the paint are not known, a wide range of parameters were chosen for reconstruction. First, this is the volumetric concentration of particles (a common parameter for all methods). The rest of the parameters were assigned in accordance with the chosen model: Mie/Rayleigh or HenyayGreenstein. In the case of the Mie/Rayleigh model, these were: particle radius and their complex refractive index (both are real and imaginary parts). For Henyay-Greenstein, the following were chosen anisotropy factor, scattering and absorption. As the objective function, measured BTDF was used, see Figure 1. Note that even though the purpose of reconstruction is ink BRDF inside the material, we used the transmission component only to speed up the calculation and also due to the fact that the reflective component contains specular peaks of the luminance factor due to Fresnel reflection of light from the substrate. The latter effect is negative for the determination of the objective function and can worsen the convergence of the optimization process and it is better to avoid this if possible.

"Simplex" method $[14,15]$ was chosen as the optimizer for all samples including sample \#1. The investigation showed it is the best method for BSDF reconstruction. The convergence for both models (Mie/Rayleigh or Henyay-Greenstein) was quite good: within 30 optimization steps, an absolute RMSD less than $0.5 \%$ was achieved. Reconstruction (RMSD calculation) was carried out for $0^{\circ}$ illumination angle only. The achieved volumetric scattering parameters are presented in the tables 1 and 2.

Table 1

Sample \#1. Value of Volume Scattering parameters after reconstruction for Mie/Rayleigh model

\begin{tabular}{clc}
\hline Number & \multicolumn{1}{c}{ Parameter Name } & Value \\
\hline 1 & Particle radius & $231 \mathrm{~nm}$ \\
2 & Particle Volume Concentration (PVC) & $5.15 \%$ \\
3 & Particle refraction index (real part) & 2.1076 \\
4 & Absorption (imaginary part of refraction index) & 0.000134
\end{tabular}

Achieved RMSD $=0.269 \%$ (RMSD between measured and reconstructed angular intensity transmitted through sample \#1). It was calculated according to (2) formula.

Table 2

Sample \#1. Value of Volume Scattering parameters after reconstruction for Henyay-Greenstein model

\begin{tabular}{ccc}
\hline Number & Parameter Name & Value \\
\hline 1 & Anisotropy factor & 0.35
\end{tabular}


Achieved RMSD $=0.237 \%$ (RMSD between measured and reconstructed angular intensity transmitted through sample \#1)

The numerical comparison of angular intensity for measured and reconstructed BSDF for Mie/Rayleigh model are presented on plots in the Figure 11.

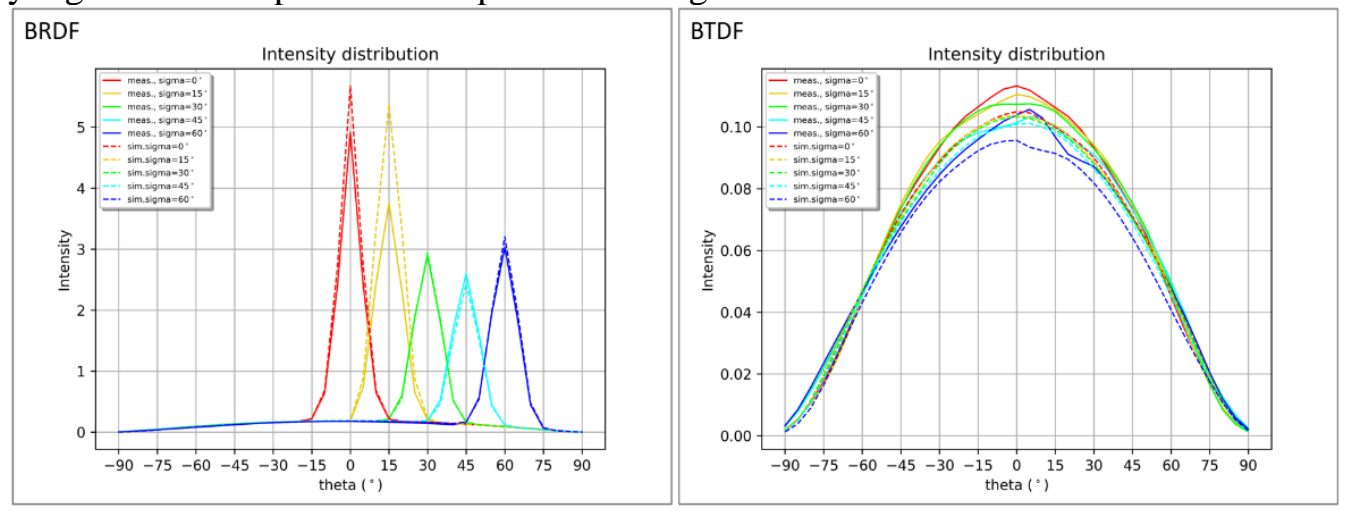

Figure 11: Sample \# 1. Comparison of angular intensity for reconstructed and measured BSDF for Mie/Rayleigh model.

Figure 11 contains plots comparing angular reflection and transmittance (angular distribution of luminous intensity) for sample \# 1 for measured and reconstructed BSDF. In the case of measurements, the sample was modeled as a single-layer surface with the assignment of the measured BSDF. In the case of the reconstructed BSDF, the model for the calculation was real with two-layers (solid) with the assignment of BSDF to one of the surfaces (upper) when the lower one was illuminated with parallel light at different angles $\left(0^{\circ}, 15^{\circ}, 30^{\circ}, 45^{\circ}, 60^{\circ}\right)$. Simulated data are showed with dashed lines and measured with solid ones. "BRDF" shows angular intensity of reflected light and "BRTD" - angular transmitted with the sample.

Figure 12 is similar to the previous one but presents Henyay-Greenstein model of Volume Scattering.

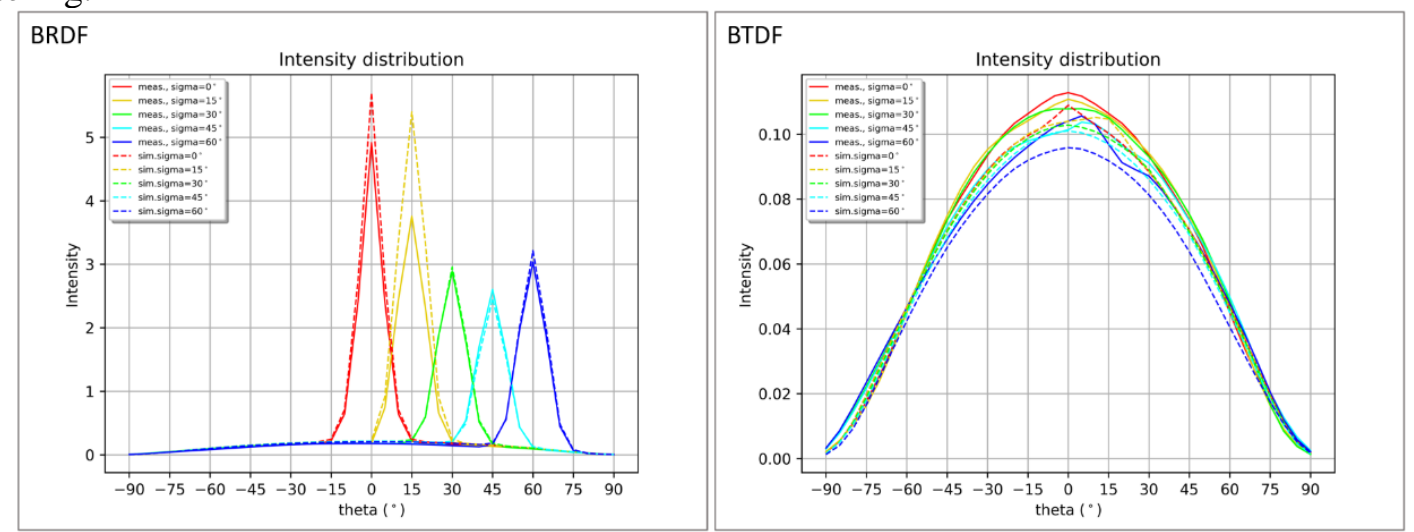

Figure 12: Sample \#1 Comparison of angular intensity for reconstructed and measured BSDF for Henyay-Greenstein model.

The images of figure 13 presents visualization of measured and simulated ink on test samples. Note the explanation about models used for images generation was done in 4.2 chapter. 


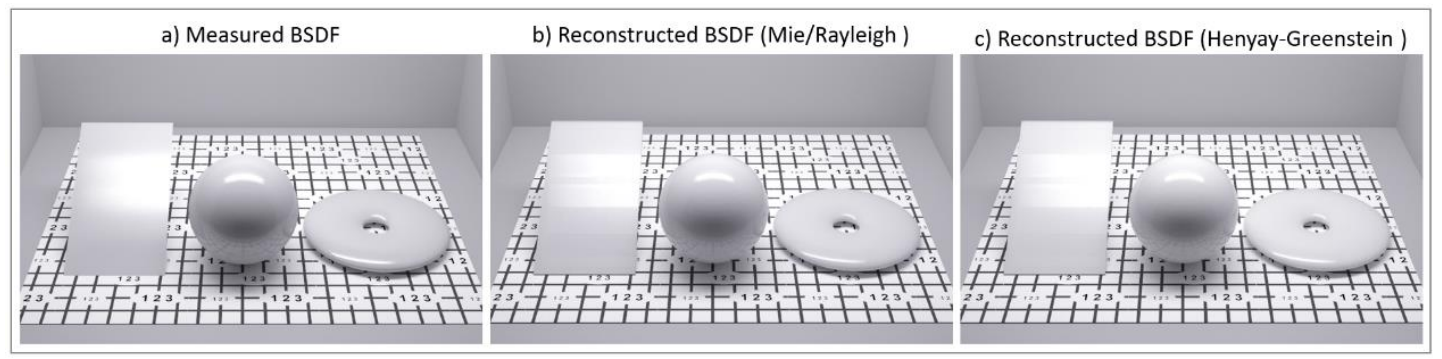

Figure 13: Sample \#1 Images generated for measured and reconstructed BSDFs

\subsection{Results of sample \#2 reconstruction}

There is a roughness on the boundary "ink-air" in the sample \#2. Note that this should not strongly affect the reconstructed BSDF, since it must be determined from the side of the substratum, but the roughness has to be considered in the reconstruction process, because it affects the measured BSDF of the entire sample. This significantly complicates the reconstruction process due to the increase in the set of parameters for optimization. In the case of the Mie/Rayleigh model, the following parameters were chosen for reconstruction: the volume concentration of particles, absorption (the imaginary part of the refractive index of the particles), and the real part of the refraction of particles. The rest of the ink parameters were selected based on trial reconstruction attempts. To optimize the roughness, the numerical method "Normals" (as the simplest) with the "Cauchy" distribution function and "sigma" angular parameter for "Cauchy" function was chosen [9]. For the Henyay-Greenstein method, Scattering and Absorption were used instead of the refractive indices of the particles. BSDF of transmitted light was selected as the objective function. Note as in the case of sample \#1 it was measured for the entire sample with ink and illuminated from the side of the substrate. "Simplex" method was chosen as the optimizer for sample \# 2 also. The convergence for both models (Mie/ Rayleigh and Henyay-Greenstein) was rather good in case \# 2 of the sample: within 30-40 optimization steps, an absolute RMSD within $1 \%$ was achieved. Reconstruction (RMSD calculation) was carried out for $0^{\circ}$ illumination angle only. The obtained parameters of volume scattering and roughness are presented in the 3 and 4 tables.

\section{Table 3}

Sample \# 2. Value of Volume Scattering parameters after reconstruction for Mie/Rayleigh model

\begin{tabular}{clc} 
Number & \multicolumn{1}{c}{ Parameter Name } & Value \\
\hline 1 & Particle radius & $250.0 \mathrm{~nm}$ \\
2 & Particle Volume Concentration (PVC) & $1.77 \%$ \\
3 & Particle refraction index (real part) & 1.8 \\
4 & Absorption (imaginary part of refraction index) & 0.001 \\
5 & "Sigma" angle (for "Cauchy" function, "Normals method") & $3.2^{\circ}$ \\
\hline
\end{tabular}

Achieved RMSD $=0.907 \%$

Table 4

Sample \#2. Value of Volume Scattering parameters after reconstruction for Henyay-Greenstein model

\begin{tabular}{clc}
\hline Number & \multicolumn{1}{c}{ Parameter Name } & Value \\
\hline 1 & Anisotropy factor & 0.57 \\
2 & Particle Volume Concentration (PVC) & $1.97 \%$ \\
3 & Scattering & 26.6 \\
4 & Absorption & 0.0001 \\
5 & "Sigma" angle (for "Cauchy" function, "Normals method") & $2.9^{\circ}$ \\
\hline
\end{tabular}

Achieved RMSD = 1.231\% 
The numerical comparison of angular intensity for measured and reconstructed BSDF for Mie/Rayleigh model are presented on plots in Figure 14 and for Henyay-Greenstein method in Figure 15. The all plots are similar to Figures 11 and 12 for sample \#1
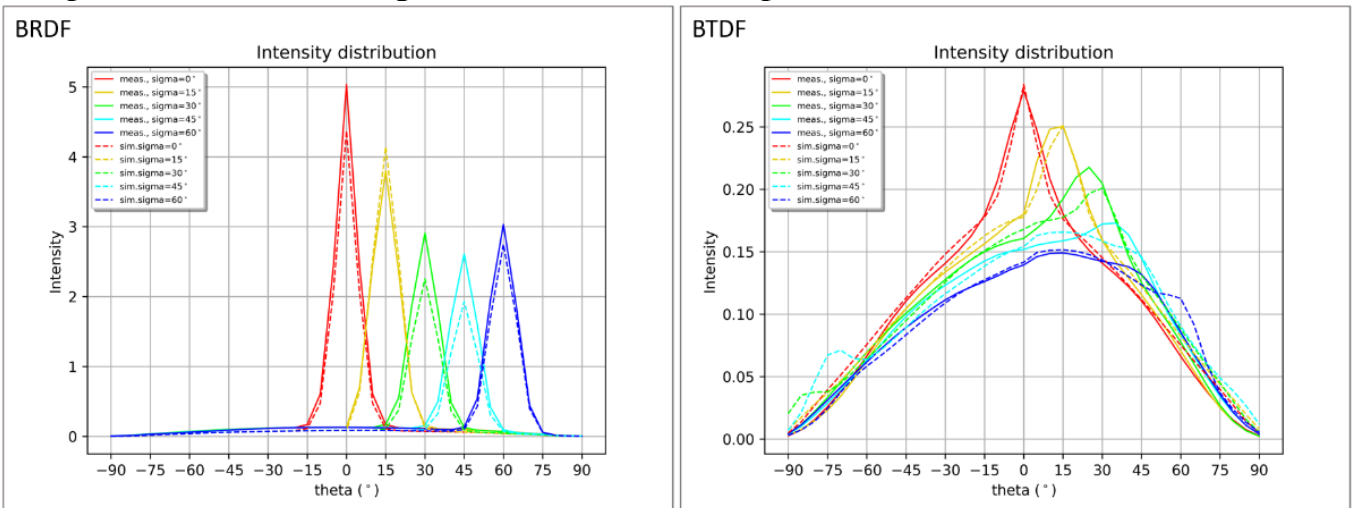

Figure 14: Sample \# 2. Comparison of angular intensity for reconstructed and measured BSDF for Mie/Rayleigh model.
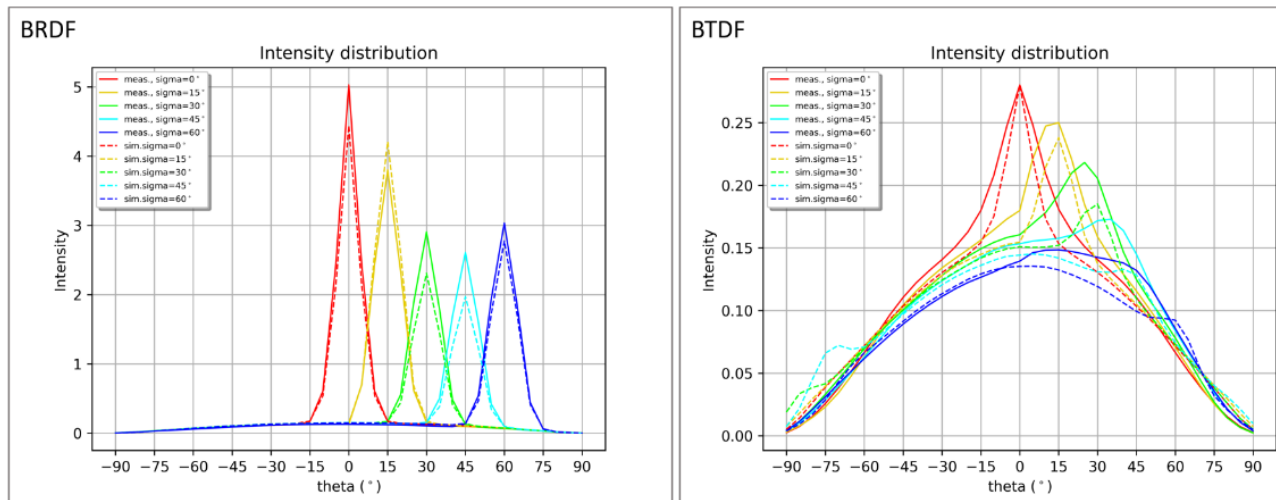

Figure 15: Sample \#2 Comparison of angular intensity for reconstructed and measured BSDF for Henyay-Greenstein model.

Visualization of sample \#2 ink is shown in Figure 16.

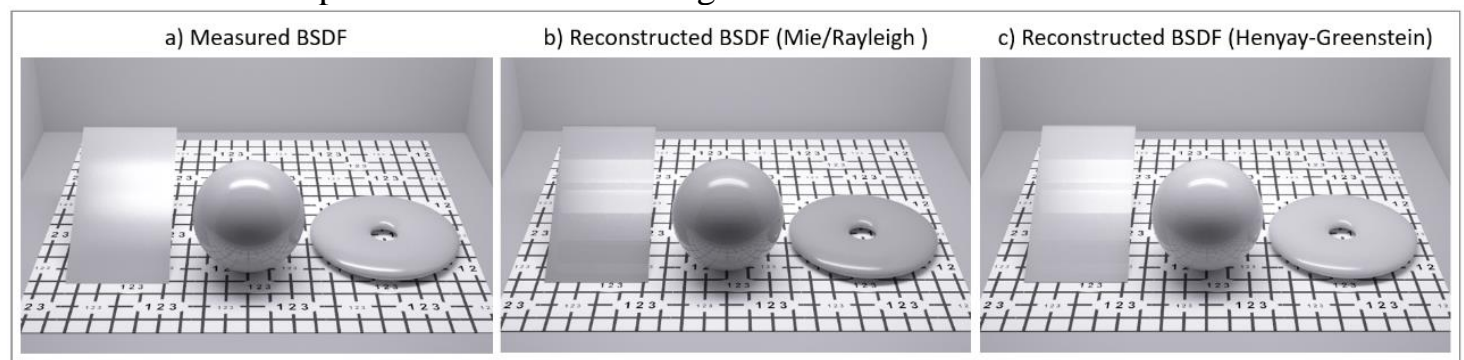

Figure 16: Sample \#2 Images generated for measured and reconstructed BSDFs

\subsection{Results of sample \#3 reconstruction}

BSDF reconstruction for sample \# 3 is similar to \# 1, \# 2 with one small but noticeable difference the ink is colored, and color reproduction is required. This is a serious complication, since many ink parameters affect the reproduction of light color and this characteristic is very sensitive. Ideally, each parameter of volume scattering should be modeled as a spectrogram, however there is no necessary measured data for this, and it is practically impossible to add color (spectrograms) to the optimization process of BSDF reconstructing due to the catastrophic increase in the parameters for optimization. To somehow solve this problem, the following approach was used:

1. In the measured BRDF the spectral distribution of the integral coefficient of diffuse reflection (averaged over all directions of illumination) was extracted. It is marked as $K_{\mathrm{d}}(\lambda)$. 
2. The further process depends on the volume scattering model. In the case of the Mie/Rayleigh model, this data can be used as a spectrogram for particle absorption (imaginary refractive index). In accordance with the theory, the imaginary refractive index connects the energy of the input $I_{o}(\lambda)$ and transformed (reflected, transmitted) light $I(\lambda)$ by (4) formula:

$$
I(\lambda)=I_{o}(\lambda) e^{\frac{-4 \pi d i(\lambda)}{\lambda}}
$$

where $i$ is the imaginary part of the refractive index and $d$ is a distance (some constant value can be set in our case). Using the above characteristics, it is possible to link it with the integral reflectance of the input and reflected light:

$$
K_{d}(\lambda)=\frac{I(\lambda)}{I_{o}(\lambda)}
$$

Substituting (5) into (4), one can easily find the spectrogram for the imaginary part of the refractive index:

$$
i(\lambda)=-\frac{\lambda \ln \left(K_{d}(\lambda)\right)}{4 \pi d}
$$

The calculated absorption spectrogram (imaginary refractive index) is used in the optimization process of reconstruction by modifying its average (over all wavelengths) value. In other words, the shape of the spectrogram does not change during BSDF reconstruction process. For Henyay-Greenstein volume scattering model the similar approach is used with one difference: the integral reflectance spectrogram $K_{\mathrm{d}}(\lambda)$ is plugged directly into the Scattering parameter and the mean value of the spectrogram is optimized.

BSDF for sample \# 3 is measured from the substratum side and contains luminance peaks due to Fresnel reflection. In order to reduce their parasitic influence on the optimization process, the reflection functions calculated for the measured and simulated BSDFs are converted to a logarithmic scale and then the relative RMSD (defined with (3) formula) between them is calculated and it is an objective optimization function. The calculation was carried out on a limited spectral range of 570-630nm, which is justified since, according to the input characteristics, the lighting system of the device, where this ink is used, operates in this limited range. The "Simplex" method [14] was chosen as the optimizer for sample \#3 similar to other samples. Optimization convergence for both models (Mie/Rayleigh and Henyay-Greenstein) was not perfect. The achieved RMSD was about 10\% and further optimization does not improve the output. Not ideal recovery results can be explained by simplifications adopted for the recovery process. This may be the goal of further deeper research in this area. The achieved volumetric scattering parameters for sample \#3 are presented in the tables 5 and 6

\section{Table 5}

Sample \#3. Value of Volume Scattering parameters after reconstruction for Mie/Rayleigh model

\begin{tabular}{clc}
\hline Number & \multicolumn{1}{c}{ Parameter Name } & Value \\
\hline 1 & Particle radius & $250 \mathrm{~nm}$ \\
2 & Particle Volume Concentration (PVC) & $14.0 \%$ \\
3 & Particle refraction index (real part) & 1.8 \\
4 & Absorption (imaginary part of refraction index) & $0.0001^{*}$ \\
\hline
\end{tabular}

Achieved RMSD $=13.7 \%$ (RMSD between measured and reconstructed angular intensity reflected on sample \#3). It was calculated according to (3) formula and for all wave lengths used in optimization process.

Table 6

Sample \#3. Value of Volume Scattering parameters after reconstruction for Henyay-Greenstein model

\begin{tabular}{clc}
\hline Number & \multicolumn{1}{c}{ Parameter Name } & Value \\
\hline 1 & Anisotropy factor & -0.57 \\
2 & Particle Volume Concentration (PVC) & $31.87 \%$ \\
3 & Scattering & $64.95^{*}$ \\
4 & Absorption & 0.0052 \\
\hline
\end{tabular}

Achieved RMSD $=12.1 \%$

Note the parameters of the substrate material (thickness, refractive index) were set constant in accordance with the input sample specification presented in chapter 5 and did not participate in the 
BSDF reconstruction process. The thickness of the paint layer was set $=100 \mu \mathrm{m}$. " "* marks the values represented by the average values of the spectrograms

The numerical comparison of angular intensity for measured and reconstructed BSDF for both Mie/Rayleigh and models is presented on plots in Figure 17. Only one BSDF component of reflected light (BRDF) is presented.
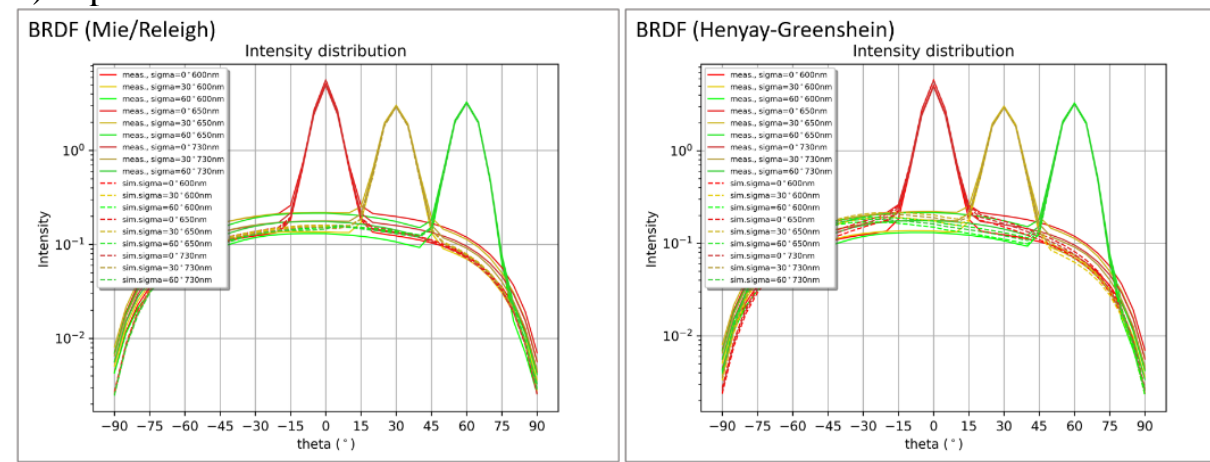

Figure 17: Sample \# 3. Comparison of angular intensity for reconstructed and measured BRDF for Mie/Rayleigh and Henyay-Greenstain models.

Plots in Figure 17 presents angular intensity in spectral format (for 3 wave lengths $-600 \mathrm{~nm}, 650 \mathrm{~nm}$, $730 \mathrm{~nm})$.

Visualization of sample \#3 is presented in Figure 18.

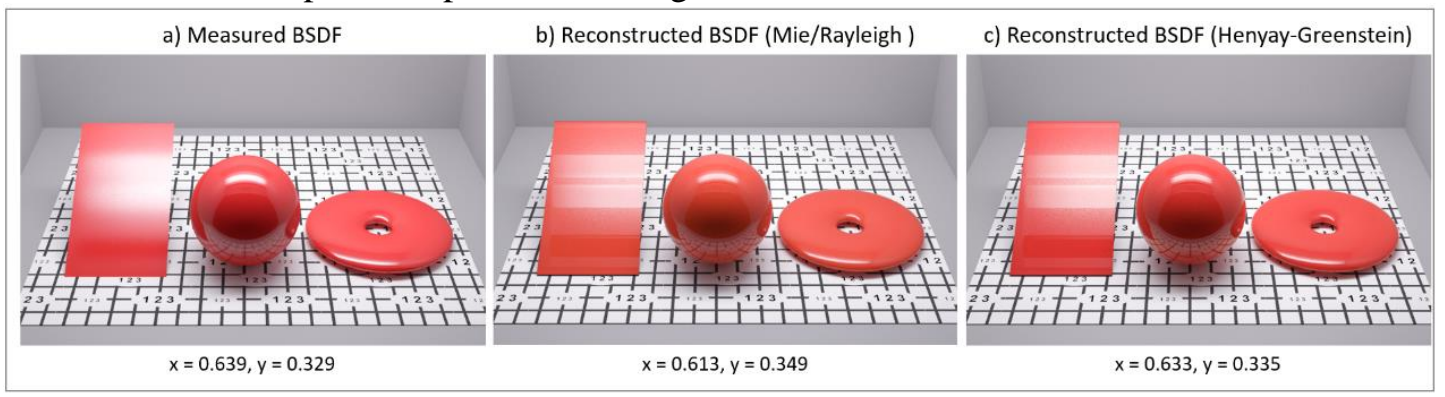

Figure 18: Sample \#2 Images generated for measured and reconstructed BSDFs

Note $\mathrm{x}, \mathrm{y}$ in Figure 18 are chromaticity values taken for the center of sphere test object.

\section{Conclusions}

Reconstruction of all three real samples covered by ink shows good agreement with measurements results. Firstly, it is seen on a quantitative level comparing plots with reconstructed BSDF versus BSDF obtained with direct measurements. Secondly, it is proved with the visual evaluation of generated images for test objects with ink where we can see close similarity in shape of highlight zones, absence of any artifacts and color reproduction of colored sample. Both methods of the phase function of scattering agents presentation (Henyay-Greenstein and Mie/Rayleigh) are investigated. In the case of sample \#1, both approached give practically the same good output. In the case of sample \#2 with microroughness, Mie/Rayleigh method showed better agreement with measurements while in the case of sample \#3 direct specification of spectral scattering available in the Henyay-Greenstein approach gives better color reproduction of colored ink. So, both approaches are worth to be considered for application in the reconstruction of ink scattering properties.

\section{Acknowledgements}

The work was supported by RFBR, Grant № 20-01-00547. 


\section{References}

[1] V. G. Sokolov, D. D. Zhdanov, I. S. Potemin, B. Barladian, N. N. Bogdanov, Optimization based on reconstruction of volume scattering medium parameters, in: Proc. SPIE, volume 10693, Illumination Optics V, 1069312, 2018. doi: 10.1117/12.2312724

[2] T. A. Germer, Bidirectional scattering distribution function measurements from volume diffusers: correction factors and associated uncertainties, Applied optics 55(25) (2016) 6978-6982. doi: 10.1364/AO.55.006978

[3] S. Nurcan, E. Serkan, Ö. Aydın, BRDF Reconstruction Using Compressive Sensing, 21st International Conference on Computer Graphics, Visualization and Computer Vision, 2013, pp. 88-94, ISBN 978-80-86943-74-9

[4] J. B. Nielsen, H. W. Jensen, R. Ramamoorthi, On Optimal, Minimal BRDF Sampling for Reflectance Acquisition, ACM TOG 34(6) (2015) 1-11. doi: 10.1145/2816795.2818085

[5] K. W. Calabro, W. Cassarly, Modeling scattering in turbid media using the Gegenbauer phase function,in: Proc. SPIE 9333, Biomedical Applications of Light Scattering IX, 93330F (2015). doi: $10.1117 / 12.2079695$

[6] S. Magarill, W. J. Cassarly, D. R. Jenkins, et. al., Parametric Model of Volumetric Scattering, Design and Fabrication Congress 2017 (IODC, Freeform, OFT), OSA, 2017. doi: 10.1364/IODC.2017.ITh3A.4

[7] Q. Kuperman-Le Bihan, Volume scattering characterization for illumination design, in: Proc. SPIE 8550, Optical Systems Design, 85502K (2012). doi: 10.1117/12.981995

[8] Lumicept - Hybrid Light Simulation Software, URL: http://www.integra.jp/en

[9] V. G. Sokolov, D. D Zhdanov, I. S. Potemin, N. N. Bogdanov, A. D. Zhdanov, E. U. Denisov, Reconstruction of BSDF based on optimization of Micro-Relief normal distribution, in: Proc. of 27th International Conference on Computer Graphics and Vision, 2017, pp. 37-41.

[10] I. Renhorn, G. D. Boreman, Analitical fitting model for rough-surface BRDF, Optics Express 16(17) (2008) 12982-12898. doi: 10.1364/OE.16.012892

[11] V. Sokolov, D. Zhdanov, I. Potemin, A. Zhdanov, N. Deryabin, A Bidirectional Scattering Function Reconstruction Method Based on Optimization of Microrelief Heights Distribution, in: CEUR Workshop Proceedings, volume 2744, 2020. doi: 10.51130/graphicon-2020-2-3-6

[12] Muracami Color Research Laboratory, URL: http://www.mcrl.co.jp/english/index.html

[13] D. D. Zhdanov, I.S. Potemin, V. G. Sokolov, A. A. Garbul, A. G. Voloboy, V. A. Galaktionov, Simulation of the BSDF measurement capabilities for various materials with GCMS-4 goniospectrophotometer, in: Proc. of SPIE, volume 10021, 2016. doi: 10.1117/12.2245739

[14] R. E. Stone, C. A. Tovey, The simplex and projective scaling algorithms as iteratively reweighted least squares methods, SIAM Review 33 (2) (1991) 220-237. doi:10.1137/1033049

[15] T. Hansen, U. Zwik, An improved version of The Random Facet Pivoting Rule for the Simplex Algorithm, in: Proceeding of forty-seventh ACM symposium on Theory of Computing (STOC '15), New-York, NY, USA, 2015, pp. 209-218. doi: 10.1145/2746539.274655 